patient leads to a decrease in bone mass. The bone loss greater is observed in the 6 months after the spinal cord injury, and stabilizing between 12-16 months after the same. The incidence of fractures oscillates between $1.5 \%$ and $6 \%$.

Objectives: To assess bone metabolism and bone fracture incidence in SPI patients.

Methods: Prospective study of SPI patients from the Spinal Cord Injury Unit of La Fe Hospital. In all cases densitometry, x-ray image, bone metabolism biomarkers and clinical evaluation have been performed according protocol. Statistical techniques was carried out using $R$ software 3.2.3, using mixed linear regression models.

Results: We studied 40 patients with SCI, $54 \%$ of them men and $46 \%$ women, with a mean age of 59.5 years (57.3- 63.5). The $58 \%$ of patients showed thoracic injuries, $48.6 \%$ paraparesis and $46.7 \%$ presented level C in Asia scale. The baseline study was performed in $100 \%$ of patients $(n=40), 65 \%$ in month $6(n=26)$, $30 \%$ in month $12(n=12)$, and in $22.5 \%$ in month $18(n=9)$. The $32.4 \%$ of patients received supplementation with calcium and vitamin $D$ at month $0,66.7 \%$ at month 6 and $100 \%$ at month 12 .

In month 6 , the $11.1 \%$ was treated with antiresorptive drugs.

An increase in vitamin $D$ values can be observed in the population with follow-up (values of 16.82 in month 0 to 39.33 in month 12), justified by the supplementation, and there is an increase in Calcium and a decrease in phosphorum values.

There was also a decrease in PTH levels in month 12 (32.3) compared to month 0 (34.08), as well as a decrease in bctx levels. Probably related to the increase of vitamin D.

Despite a decrease in the densitometric parameters at month 6, a slight recovery in bone mineral density at month 12 was observed. No bone fractures were seen during Follow-up in none of the patients.

Results from biochemical markers and densitometry are showed in the table

\begin{tabular}{lccc}
\hline Parameter & Month 0 & Month 6 & Month 12 \\
\hline & Mean (SD) & Mean (SD) & Mean (SD) \\
Calcium & $9.04(0.47)$ & $9.57(0.36)^{\star \star \star}$ & $9.39(0.34)^{\star \star \star}$ \\
Phosphorus & $3.83(0.62)$ & $3.42(0.45)^{\star \star \star}$ & $3.4(19.94)^{\star \star \star}$ \\
25OH Vitamin D & $16.82(10.4)$ & $37.27(15.52)^{\star \star \star}$ & $39.33(19.94)^{\star \star \star}$ \\
PTH & $34.08(21.74)$ & $36.16(19.46)$ & $32.3(12.6)$ \\
P1NP & $76.1(79.18)$ & $64.27(28.11)$ & $65.87(25.76)$ \\
BCTX & $0.94(0.46)$ & $0.62(0.87)^{\star \star}$ & $0.34(0.21)^{\star \star \star}$ \\
Lumbar spine tscore & $-0.24(1.58)$ & $-0.25(1.11)$ & $-0.3(1.27)$ \\
Femoral neck tscore & $-0.73(1.34)$ & $-1.35(1.03)^{\star \star}$ & $-1.26(1.25)^{\star \star}$ \\
Hip bone tscore & $-0.74(1.25)$ & $-1.56(1.29)^{\star \star \star}$ & $-1.52(1.4)^{\star \star \star}$ \\
\hline${ }^{*}$ ( & & &
\end{tabular}

${ }^{*} \mathrm{p}<0,05,{ }^{* *} \mathrm{p}<0,01,{ }^{* * *} \mathrm{p}<0,001$.

Conclusions: A high percentage of our patients with spinal cord injury has a vitamin $D$ deficiency. In addition, the lower levels are associated with cases where the mobility limitation is higher. As patients increase vitamin D values, a decrease in the bctx and PTH parameters is observed.

No fractures were detected during follow-up.

Disclosure of Interest: None declared

DOI: 10.1136/annrheumdis-2017-eular.5487

\section{AB0838 EPIDEMIOLOGY AND ANALYSIS OF FALLS IN PATIENTS DURING BALNEOTHERAPY IN DANUBIUS HEALTH SPA PIESTANY}

I. Rybar $^{1}$, V. Kolnikova ${ }^{2}$, T. Zimanova ${ }^{2}$, R. Gaspar ${ }^{2} .{ }^{1}$ Department of Rheumatology, National Institute of Rheumatic Diseases; ${ }^{2}$ Department of Physiotherapy, Danubius Health Spa, Piestany, Slovakia

Background: Patients falls in hospitals are frequent and undesirable complications that may lead to negative outcomes such as injuries, prolonged hospitalization and legal liability.

Objectives: To investigate the incidence and characteristics of patients hospitalized in the Health Spa Piestany.

Methods: Prospective analysis of falls in the group of in-patients of health spa during a year since 01.01 .2015 to 31.12 .2015 .

Results: Overall there were hospitalized in the health spa 25774 in-patients. The mean age of patients was $64,02 \pm 13,16$ years. There were women - 15301 , mean age $(63,61 \pm 11,57$ years) and men 10473 , with mean age $(64,43 \pm 9,22$ years). Falls were confirmed in 131 patients (women 94, mean age 68,55 $\pm 10,22$ years and men 37 males, mean age 70,39 $\pm 7,62$ years. Fractures have been confirmed in 19 patients, all of them non-vertebral. The ankle distortion were in 11 patients and lacerated wounds in 10 patients. There were collapse status in 8 and commotio cerebri in 1 patient. Spa therapy had to be discontinued only in 5 from 131 patiens due to falls. The annual incidence of falls reached 5,08 cases/1000 patients. In women there were - 6,14 cases/1000 women a year and in men 3,53 cases/1000 men a year. The annually incidence of fractures reached $0,69 / 1000$ pacients. The most common locations of falls were hotel rooms, especially bathtub in the bathroom (49\%), slightly less facilities for balneotherapy $(22 \%)$, the area outside the spa $(21 \%)$ and at least in the park area of health spa $8 \%$. Women and men who have suffered falls had significantly higher age and had also high rate of cardio-vascular morbidity (87\%).

Conclusions: The annual incidence of falls among patients in a balneotherapy reached to 5.08 cases per 1,000 patients per year. Incidence of falls in women was almost twice as high compared to men. The age of women and men with falls was significantly higher $(\mathrm{p}<0,001)$ compared to other patients and they also had higher rate of cardiovascular morbidity. The most common locations of falls were bathtubs in the bathroom.

References:

[1] Gazibara T. et al.: Psychogeriatrics. 2017 Jan 27. doi: 10.1111/psyg.12217.

[2] Cockayne S. et al.: PLoS One. 2017 Jan 20;12(1):e0168712.

[3] Tsonga T. et al.: Clin Orthop Surg. 2015 Dec;7(4):449-56.

Disclosure of Interest: None declared

DOI: 10.1136/annrheumdis-2017-eular.5278

\section{AB0839 THE EFFICACY AND SAFETY OF DENOSUMAB LOCAL EXPERIENCE)}

J. Al-Saleh, N. Salah, A.S. Hasan, S. Mohamad, F.A. ElBadawi, M. Khamashta. Rheumatology Department, Dubai Hospital, Dubai, United Arab Emirates

Background: Denosumab was introduce to the United Arab Emirates Market in 2013.Given the limited experience in using Denosumab in the region we have explored it efficacy and safety in daily practice.

Objectives: To assess the efficacy and safety of Denosumab in our practice.

Methods: Inclusion criteria: All patients received Denosumab in a dose of $60 \mathrm{mg}$ every six months. Underwent DEXA scan in Dubai Health Authority. Exclusion from analysis: Other Doses of Denosumab, who received less than 3 doses, and those with no follow up DEXA scan. Outcome measures: 1) Efficacy: Proposed Criteria for Assessing Clinical Response $[1,2]$. a) "inadequate": incident fracture and significant BMD decrease. b) "possible inadequate": incident fracture or significant BMD decrease. c) "appropriate": no fracture and stability or increase in BMD. 2) Safety: Reviewing the medical records and conduct patients interview for occurrence of the following adverse events. Statistical analysis: Descriptive statistical analysis, Graphpad Prism 6 was used.

Results: 143 patient identified. Out 139 patients 86 were eligible for analysis (See table 1). At baseline $39 \%$ had normal vitamin D level, $57 \%$ had insufficiency and $4 \%$ had deficiency. 20 of 86 did not undergo repeated DEXA scan. $9 \%$ had osteopenia and $91 \%$ had osteoporosis before initiating Denosumab in comparison $8 \%$ had normal bone mineral density, $45 \%$ had osteopenia and $47 \%$ post four injections of Denosumab. Table 2 summarize the comparison between the responders and non-responders. There was a significant positive correlation in the increase in bone mineral density among the responders at the femoral neck and the lumbar spine, $(r=0.56,95 \%$ confidence interval: $0.31-0.74, \mathrm{P}$-value $<0.0001$ No report of any of the adverse events 86 patients who completed 2 years or more on treatment.

Table 1. Patients characteristics at baseline

\begin{tabular}{lcc}
\hline Variables & Number & Percentage \\
\hline Age (yrs) median, (1st Quartile- 3rd Quartile) & $65,(58-67)$ & \\
$51-65$ & 48 & $35 \%$ \\
$>65$ & 76 & $55 \%$ \\
Female & 126 & $91 \%$ \\
UAE & 121 & $87 \%$ \\
Comorbidities & 72 & $52 \%$ \\
Received 4 Denosumab doses or more & 86 & $62 \%$ \\
DEXA scan in DHA at Baseline & 136 & $98 \%$ \\
Osteopenia & 12 & $9 \%$ \\
Osteoporosis & 124 & $91 \%$ \\
Fractures & 12 & $9 \%$ \\
\hline
\end{tabular}

Table 2. Comparison between the appropriate Response group "Responders" and inadequate response group "Non-responders"

\begin{tabular}{lccccc}
\hline Variables & Responders & Non-responders & Odds Ratio & P-value & $95 \% \mathrm{Cl}$ \\
\hline Number & 52 & 14 & & & \\
Rheumatic Diseases & 6 & 36 & 0.1 & 0.0001 & $0.05-0.2$ \\
Fracture & 0 & 3 & 0.2 & NS & \\
Pre-treatment fractures & 9 & 1 & 9.5 & 0.02 & $2.0-70$ \\
\hline
\end{tabular}

Conclusions: Denosumab was effective and safe in our patients. Long-term follow up is required to verify these findings in our population.

References:

[1] Diez-Perez and Gonzalez-Macias. Inadequate responders to osteoporosis treatment: proposal for an operational definition. Osteoporos Int. 2008 Nov;19(11):1511-6.

[2] E. M. Lewiecki \& N. B. Watts. Assessing response to osteoporosis therapy Osteoporos Int (2008) 19:1363-1368.

Disclosure of Interest: None declared

DOI: 10.1136/annrheumdis-2017-eular.6791 